\title{
Review of the Afrotropical species of the genus Prometopia (Coleoptera: Nitidulidae: Prometopiinae)
}

\author{
Josef JELÍNEK \& Jiř́i HÁJEK \\ Department of Entomology, National Museum, Cirkusová 1740, CZ-193 00 Praha 9 - Horní Počernice, Czech Republic; \\ e-mails:jj.nitidula@seznam.cz; jiri_hajek@nm.cz
}

\author{
Accepted: \\ $19^{\text {th }}$ March 2020 \\ Published online: \\ $27^{\text {th }}$ March 2020
}

\begin{abstract}
African species of the genus Prometopia Erichson, 1843 are reviewed. Two new species: Prometopia hirta sp. nov. (Zambia and Tanzania) and P. intermedia sp. nov. (Tanzania) are described and illustrated. Two previously known Afrotropical species, Prometopia binotata Murray, 1867 and Prometopia quadrinotata Grouvelle, 1896 are redescribed, and a key to Afrotropical species of Prometopia is given as well as new data on their distribution. Notes to correct the spelling of the subfamily Prometopiinae is provided.
\end{abstract}

Key words. Coleoptera, Nitidulidae, Prometopia, taxonomy, new species, new records, key, Tanzania, Zambia, Afrotropical Region

Zoobank: http://zoobank.org/urn:1sid:zoobank.org:pub:ADACFB39-2CB6-4969-8B23-612307C875B2

C 2020 The Authors. This work is licensed under the Creative Commons Attribution-NonCommercial-NoDerivs 3.0 Licence.

\section{Introduction}

Prometopia Erichson, 1843 is a nominotypical genus of the recently reinstituted subfamily Prometopiinae (CLINE et al. 2014), previously defined as an informal 'Axyra complex' of genera by AUDisio \& JELÍNEK (1993). It currently contains 28 species distributed all over the world except for most of Palaearctic Region, Madagascar, and New Zealand. This count does not included species of the closely related Oriental Parametopia Reitter, 1884, currently classified either as the subgenus of Prometopia (KIREJTSHUK 2005, 2008) or, traditionally, as a distinct genus (JelíneK \& Audisio 2007, Cline et al. 2014). Two species of Prometopia are currently known from the Afrotropical Region: Prometopia binotata Murray, 1867 and Prometopia quadrinotata Grouvelle, 1896, both subsequently mentioned only in the catalogue (Grouvelle 1913). Two new species: Prometopia hirta sp. nov. from Zambia and Tanzania, and $P$. intermedia sp. nov. from Tanzania are described in the present paper, along with redescriptions of other African species, and a key to their identification is provided.

\section{Material and methods}

Examination, dissection and measurements were completed with the use of an Olympus SZX7 stereomicroscope with an ocular micrometer. Body length was measured from the anterior margin of the clypeus to the apex of the elytra, and body width as the maximum width of the elytra combined.

Habitus photographs were taken using a Canon EOS 550D digital camera with an attached Canon MP-E65mm $\mathrm{f} / 2.81-5 \times$ macro lens as numerous separate images at different focal planes and subsequently combined using Helicon Focus 6.3.0 software. The male genitalia were studied and illustrated in temporary glycerine mounts using an Olympus BX41 transmitted light microscope with Canon DS126291 attachment; they were subsequently washed in distilled water and mounted in euparal on the same card as the beetle.

The following acronyms are used for morphological terms:
ANCL length of antennal club;
ANCW width of antennal club;
ANLE length of antenna;
HEAW width of head across eyes;
LELY length of elytra from the tip of scutellar shield to the tip of elytra;
LEPR length of pronotum along median axis;
LEV1 length of abdominal ventrite I along median axis;
LMTV length of metaventrite along median axis;
WELY maximum width of elytra combined;
WPR1 width of pronotum between posterior angles;
WPR2 maximum width of pronotum;
WPR3 width of pronotum between anterior angles. 
Exact label data are cited for the type material. Individual labels are separated by a double slash (//), different rows by simple slash (/). Additional comments and/or explanatory notes are given in square brackets and the following abbreviation is used: $\mathrm{p}$ - printed.

Specimens included in this paper are deposited in the following institutional or private collections:

ALBC Andrzej Lasoń collection, Bialystok, Poland;

BMNH The Natural History Museum, London, United Kingdom [former British Museum (Natural History)];

CEREB Centre d'Etudes et de Recherches Entomologiques Béninois, La Brillanne, France;

HNHM Hungarian Natural History Museum, Budapest, Hungary;

NMPC National Museum, Prague, Czech Republic;

SMNS Staatlisches Museum für Naturkunde, Stuttgart, Germany.

The distribution map was created based on the map available from Wikimedia Commons (https://commons. wikimedia.org/wiki/File:Africa_relief_location_map.jpg), created by Eric Gaba - Wikimedia Commons user: Sting.

\section{Taxonomy}

\section{Subfamily Prometopiinae}

Two original spellings of the subfamily occur in BövING \& Craighead (1931): Prometopinae in the Key (p. 37); and Prometopiinae in the Conspectus (p. 74), captions to Plate 35 (p. 156) and Index (p. 346). When reinstituting the subfamily, Curne et al (2014) used the variant Prometopinae without any comments, however since then both spellings have been used in the literature: Prometopinae (DASGUPTA et al. 2015, Jelínek \& Hájek 2018, Lee et al. 2018), Prometopiinae (KIREJTSHUK \& MANTIČ 2015, Gimmel \& FerRo 2018), or both (LAWRENCE 2016). However, Bouchard et al. (2011) had already pointed out that spelling Prometopinae is incorrect as the stem is Prometopi-. Therefore, following the ICZN (1999: Article 32.5.3.1) the correct spelling of Prometopiinae has to be used.

\section{Prometopia Erichson, 1843}

Prometopia Erichson, 1843: 279. Type species: Nitidula sexmaculata Say, 1825 (subsequent designation by LuCAs 1920).

Diagnosis. Body oblong oval to broadly oval, more or less convex. Punctation of dorsum diffuse, sometimes intermixed with larger umbillicate punctures. Pubescence variable, sometimes finer setae intermixed with longer and/or stronger setae, sometimes pubescence fine, short and almost indistinct. Head across eyes nearly as wide as distance between anterior pronotal corners, temples moderately converging posteriad. Frons as a rule concave, frontoclypeal sulcus absent, occipital sulcus deep. Antennae with 11 antennomeres, with symmetrical, trimerous, somewhat loose club; antennomere III ca. as long as IV-VI combined. Labrum transverse to semicircular with small apical excision. Mandibles in distal half almost straight with acute, shallowly bifid tips and additional acute tooth posteriorly. Terminal palpomeres of both maxillary and labial palpus elongate, subcylindrical. Mentum large, gradually narrowed distad. Pronotum transverse, anterior margin deeply trapezoidally emarginate, anterior angles sharp, prominent. Elytra simultaneously rounded apically, diffusely punctate. Prosternal process longitudinally almost flat, its apical portion strongly dilated, subtriangular, subtruncate apically. Mesoventrite simple, situated almost at the same level as metaventrite. Metacoxal lines diverging from posterior margin of metacoxal cavity both at inner and outer ends, delimiting a characteristic subtriangular space on abdominal ventrite I. Both meso- and metacoxae widely separated. Tibiae dorsoventrally flattened. All tarsomeres narrow; tarsal claws simple.

\section{Key to Afrotropical species of Prometopia}

1 (6) Body broadly oval, moderately convex (Figs 1-4). Mentum comparatively longer, less than $3 \times$ (2.7-2.9 $\times)$ wider than long. Pronotum widest at posterior angles, ratio WPR1/WPR2 $=0.97-1.00$. Elytra almost as long as their combined width or shorter (ratio WELY/LELY > 1), lateral margins of elytra arcuate. Oblique raised ridge on metepisterna meeting metasternopleural suture at the same point as mesocoxal line (Fig. 7).

2 (3) Each elytron with two round orange spots (Fig. 4). Elytra almost as long as their combined width, ratio WELY/LELY = 1.01-1.06 (mean 1.03). Pubescence of dorsal surface distinct, with long light semirecumbent setae. Tegmen parallel-sided in basal half, median lobe of aedeagus in apical portion strongly narrowed towards truncate apex, valves of phallotreme rounded apically (Figs 11, 14).

P. quadrinotata Grouvelle, 1896

3 (2) Each elytron with one round orange spot just before its midlength (Figs 1-3). Lateral margins of tegmen more or less arcuate, tips of valves of phallotreme acute (Figs 9-10, 12-13).

4 (5) Elytra distinctly shorter than their combined width, ratio WELY/LELY = 1.09-1.17 (mean 1.12). Pubescence of dorsal surface inconspicuous. Median lobe of aedeagus slightly narrowed apically, apex distinctly incised; valves of phallotreme with acute tips directed anterolaterally (Fig. 9).

P. binotata Murray, 1867

5 (4) Elytra almost as long as their combined width, ratio WELY/LELY = 1.01-1.09. Pubescence of dorsal surface distinct, with long light semirecumbent setae. Median lobe of aedeagus more distinctly narrowed apically, apex subtruncate; valves of phallotreme abruptly curved laterally at their apex (Fig. $10)$.

$P$. intermedia sp. nov.

6 (1) Body oblong oval, more strongly transversely convex (Fig. 5). Mentum more transverse, more than $3 \times(3.3 \times)$ wider than long. Pronotum widest in basal half, distinctly narrowed both anteriad and posteriad (ratio WPR1/WPR2 = 0.94-0.96), posterior angles obtuse. Elytra longer than their combined width, ratio WELY/LELY $=0.93-0.97$ (mean 0.95), lateral margins of elytra in basal half subparallel. Oblique raised ridge on metepisternum reaches metasternopleural suture before mesocoxal line (Fig. 8). 

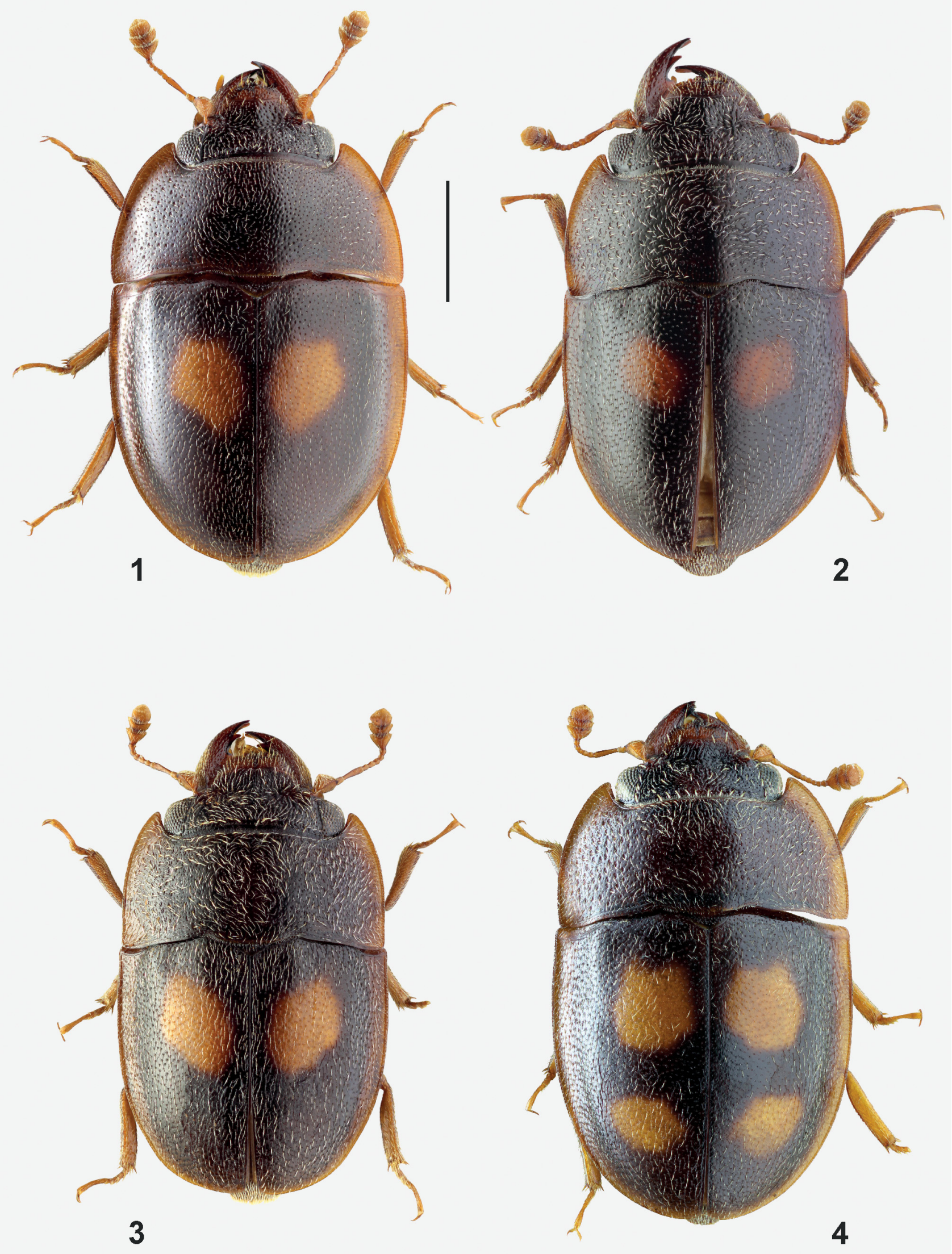

Figs 1-4. Dorsal habitus of Prometopia species. 1 - P. binotata Murray, 1867 (Zambia); 2 - P. intermedia sp. nov. ( 3 holotype); 3 - P. intermedia sp. nov. ( + ); $4-P$. quadrinotata Grouvelle, 1896 (DR Congo). Scale bar $=1 \mathrm{~mm}$. 


\section{Prometopia binotata Murray, 1867}

(Figs 1, 7, 9, 12)

Prometopia binotata Murray, 1867: 168 (original description); MuRRAY (1878): 86 (reprint of Murray 1867); Grouvelle (1913): 132 (catalogue).

Material examined.ANGOLA: I.I.A.A., Salazar, 9.-15.iii.1972, at light, 3 spec., A. G. Kirejtshuk det. 2003 (BMNH). BENIN: Zou Department, Save env., 18.-20.vi.2001, F. et L. Kantner lgt., 1 spec. (SMNS); Akonbgbere vill. near Save, 16.-20.vi. 2001, A. Kudrna jr. lgt., 1 spec. (ALCB); Bohicon, quartier Soglogon, $07^{\circ} 10^{\prime} 54^{\prime \prime} \mathrm{N}, 02^{\circ} 01^{\prime} 54^{\prime \prime} \mathrm{E}, 200 \mathrm{~m}$, 6.vi.2016, A. Coache lgt., 1 spec. (CEREB). CAMEROON: North-West Province, Bali Nyonga, 8.iii.2006, J. L.Voma lgt., 1 spec. (ALBC). CENTRAL AFRICAN REPUBLIC: Ombella-Mpoko Province, Damara-Bouca road, 95 km SW Sibut (GPS), 600 m, 24.-25.v.2009, A. Kudrna jr. lgt., 1 spec. (NMPC); Ombella-Mpoko Province, 75 km NNE Bangui, 5.-6. iv. 2010, A. Kudrna jr. lgt., 3 spec. (ALBC); Ouham-Pende Province, S Bozoum, 50 km SW Bossentele, 21.-22.iii.2010, A. Kudrna jr. lgt., 1 spec. (ALBC). EQUATORIAL GUINEA: Guinea Espaňol, Evinayong, Dr. Báguena lgt., 2 spec.; Rio Benito, Dr. Báguena lgt., 1 spec.; Mikomeseng, Dr. Báguena lgt., 1 spec. (all NMPC, ex coll. O. Marek). GHANA: 'Gold Coast', Mpraeso, 1945-1946, G. H.Thomson lgt., 1 spec., A. G.. Kirejtshuk det. 2003 (BMNH); Volta Province, WLI, Hohoe env., 3.-6. vi.2006, J. Rolčík lgt., 1 spec. (NMPC); Upper-East Province, Navrongo env., 11.-13.vi.2006, J. Rolčík lgt., 1 spec. (NMPC); Eastern region, Atewa Range, 19.-23.iii.2009, E. Kondorosy lgt., 1 spec. (HNHM); Central region, Kakum forest, $5^{\circ} 20^{\prime} 50^{\prime \prime} \mathrm{N}, 1^{\circ} 22^{\prime} 58^{\prime \prime} \mathrm{W}, 7 . i i i .2009$, E. Kondorosy lgt., 1 spec. (HNHM). IVORY COAST: Kakpin Village, Comoe O., $08^{\circ} 39^{\prime} 07.0^{\prime \prime} \mathrm{N}, 03^{\circ} 46^{\prime} 58.8^{\prime \prime} \mathrm{W}, 259 \mathrm{~m}, 27 . v i .-2 . v i i .2015$, light trap, M. Aristophanous, P. Moretto \& E. Ruzzier lgt.,1 spec.; Mt. Tonkoui Peak, $07^{\circ} 27^{\prime} 15.2^{\prime \prime} \mathrm{N}, 07^{\circ} 38^{\prime} 12.5^{\prime \prime} \mathrm{W}, 1171 \mathrm{~m}, 9 .-16 . i v .2016$, M. Aristophanous \& P. Moretto lgt., 1 spec.; same data, but 16.-21.vii.2016, 2 spec.; Yéalé Village, Mt. Nimba, $07^{\circ} 31^{\prime} 35.3^{\prime \prime} \mathrm{N}, 08^{\circ} 25^{\prime} 20.1^{\prime \prime} \mathrm{W}, 380 \mathrm{~m}$, 18.-29.iv.2016, light trap, M. Aristophanous, M. Geiser \& P. Moretto lgt., 10 spec.; same data, but 8.v.2016, 38 spec.; same data, but track before reserve border, $07^{\circ} 32^{\prime} 18.8^{\prime \prime} \mathrm{N}, 08^{\circ} 25^{\prime} 23.8^{\prime \prime} \mathrm{W}, 376 \mathrm{~m}, 18$.-29.iv.2016, 4 spec.; Mt. Nimba camp, $07^{\circ} 35^{\prime} 15^{\prime \prime} \mathrm{N}, 08^{\circ} 25^{\prime} 05^{\prime \prime} \mathrm{W}, 823 \mathrm{~m}$, 28.iv.-8.v.2016, light trap, M. Aristophanous, M. Geiser \& P. Moretto lgt., 2 spec.; Bidjan, Banco Forest (Parc National du Banco), 05 23'03.8'N, 0403'11.2" W, 39-48 m, 21.-30.v.2017, MV light, A. \& M. Aristophanous, M. Geiser \& P. Moretto lgt., 1 spec. (all BMNH). LIBERIA: Harold Olsen, Nat. Mus. Aarh., 1 spec. (NMPC, ex coll. O. Marek). MALAWI: Southern Region, Mulanje Mts., Likhubula lodge, $827 \mathrm{~m}, 15^{\circ} 56^{\prime} 15.7^{\prime \prime} \mathrm{S}, 35^{\circ} 30^{\prime} 4.9^{\prime \prime} \mathrm{E}$, 18.-25.xi.2011, P. Pacholátko lgt., 3 spec. (NMPC). NIGERIA: Ibadan, 2.iv.1957, J. L. Gregory lgt., 6 spec. (BMNH); Ibadan, iii.1965, Ř́hová lgt., 1 spec. (NMPC); near Benin, J. L. Gregory lgt., 2.-18.iv.1958, 63 spec.; same data, but 18.-30.iv.1958, 10 spec.; 19.-30.iv.1958, 23 spec.; 1.-12.v.1958, 22 spec.; 13.-19.v.1958, 17 spec.; 19.-27.v.1958, 26 spec., 20.-27.vi.1958, 11 spec. (all BMNH); Sapoba Forest near Benin, 21.iii.1972, E. W. Classey lgt., 1 spec., A. G. Kirejtshuk det. 2003 (BMNH). SIERRA LEONE: Sierra Leone, 1904-171, 1 spec., S. Endrödy-Younga det. 1968 (BMNH); Kambama village on the banks of the Moa

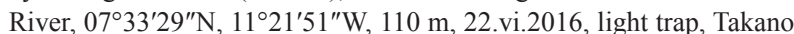
Miles \& Goff lgt., 4 spec. (BMNH); Mansonia village at the foothills of Loma Mts., $09^{\circ} 07^{\prime} 47^{\prime \prime} \mathrm{N}, 11^{\circ} 05^{\prime} 06^{\prime \prime} \mathrm{W}, 420 \mathrm{~m}$, 6.vi.2016, Takano, Miles \& Goff lgt., 5 spec. (BMNH); Loma Mountains, farmland/forest mosaic, $09^{\circ} 07^{\prime} 47^{\prime \prime} \mathrm{N}, 11^{\circ} 05^{\prime} 24^{\prime \prime} \mathrm{W}, 420 \mathrm{~m}, 11 .-15 . v i .2016$, light trap, Takano, Miles \& Goff lgt., 8 spec. (BMNH). UGANDA: Entebbe, x.1929, G. A. K. Marshall lgt., 1 spec., A. G. Kirejtshuk det. 2003 (BMNH); Kawanda, 15.ii.-6.iii.1958, at light, P. Whalley lgt., 1 spec., S. Endrödy-Younga det. 1968 (BMNH); Njala, 21.xi.1926, dead palm, E. Hargreaves lgt., 1 spec., S. Endrödy-Younga det. 1968; same data, but vi.1930, 1 spec.; 29.x.1930, 1 spec. (all BMNH). ZAMBIA: Northern province, Chipona Falls, 30 km S Chinsali, 5.-8.xii.2002, F. et L. Kantner lgt., 1 spec. (NMPC); Northwestern Province, 5 km E of Solvezi, 17.x.2008, M. Snížek lgt., 1 spec. (NMPC); Central Province, $70 \mathrm{~km} \mathrm{~N}$ Lusaka, Chisamba valley, 21.xi.2004, M. Snížek \& V. Tichý lgt., 1 spec. (ALBC); Kasanka River Pontoon, Kasanka N.P., 12³4'23"S, 30¹4'05"E, 1191 m, 2.-4.xii.2012, light trap, R. Smith \& H. Takano lgt.,1 spec. (BMNH); Kapishya Hot Springs, Shiwa N'gandu Estate, $11^{\circ} 10^{\prime} 13^{\prime \prime} \mathrm{S}, 31^{\circ} 36^{\prime} 00^{\prime \prime} \mathrm{E}, 1437 \mathrm{~m}$, 17.-21.xi.2016, light trap, Smith, Takano \& Cram lgt., 1 spec. (BMNH);

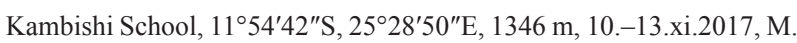
Carter, A. Lloyd, W. Miles, D. Cram \& R. Smith lgt., 1 spec. (BMNH).

Redescription. Broadly oval, moderately convex, shining. Brown to black-brown, each elytron with one round orange spot situated before midlength of elytron in sutural half. Explanate sides of pronotum and elytra, legs and antennae rusty, mouth parts red-brown. Pubescence inconspicuous (Fig. 1). Body length 3.1-4.0 mm, width 1.9-2.5 mm.

Head across eyes narrower than anterior pronotal margin. Eyes coarsely facetted, widest in anterior portion, gradually narrowed posteriad, with indistinct light interfacetal setae. Temples short, convergent. Frons separated from clypeus by transverse impression; clypeus truncate, not bordered, its surface forming obtuse angle with frons. Punctures of frons smaller than eye-facets, separated by about one diameter, becoming closer at eyes and at anterior corner of clypeus, intermixed with a few scattered larger punctures nearly equal in size to eye-facets. Interspaces smooth and shining. Antennae shorter than width of head across eyes, ratio HEAW/ANLE $=1.18-1.20$. Antennomere III almost as long as IV-VIII combined. Antennal club oval, ratio $\mathrm{ANLE} / \mathrm{ANCL}=3.25-3.33$.

Pronotum transverse (ratio WPR2/LEPR $=2.38-2.61$ ), widest at base (ratio WPR1/WPR2 $=0.97-1.00$ ), gradually narrowed anteriad, ratio WPR $1 /$ WPR $3=1.51-1.81$, WPR2/WPR3 = 1.54-1.81. Anterior margin not bordered, truncate; anterior angles acute, strongly prominent. Basal margin besides prescutellar lobe obliquely subtruncate and bordered (not bordered on lobe), basal rim with series of fine punctures. Posterior angles feebly obtuse, not prominent. Lateral margins arcuate, gradually converging anteriad, sides narrowly explanate, as wide as antennal flagellum. Punctures of rather constant size, separated by about 1 diameter, at sides intermixed with scattered larger punctures nearly equal in size to eye-facets. Pubescence consisting of short and thin dark recumbent and inconspicuous setae; sometimes intermixed with longer setae, especially at sides.

Scutellar shield transverse, almost semicircular, smooth and shining except for group of very fine dense punctures in middle.

Elytra broadly oval, regularly convex, widest in basal portion, distinctly wider than long, ratio WELY/LELY = 1.09-1.17 (mean 1.12). Lateral margins arcuate, gradually converging posteriad, slightly so anteriad; sides explanate, as wide as antennal flagellum. Humeral angle obtuse. Humeral bulges low, inconspicuous. Sutural line indistinct. Punctures like on pronotum, but generally sparser, mostly separated by more than one diameter, becoming somewhat larger laterally. Pubescence like on pronotum.

Legs. Femora oval, somewhat more than twice as long as wide. Protibia ca. $3.75 \times$ longer than wide, widest at distal end, outer subapical angle subrectangular, not prominent. Meso- and metatibia ca. $4.18 \times$ and $5.50 \times$ longer than wide respectively, flat, outer subapical angle rounded.

Ventral surface. Postmentum shallowly concave, punctate; punctures smaller than eye-facets, separated by less than one diameter, interspaces smooth and shining. Antennal furrows deep, partly concealed by extended outer edges 
of postmentum, converging posteriad, posterior to temples continued as an incised arcuate series of punctures curved outwards. Genae posterior to eyes impunctate. Prosternum broadly transversely vaulted, punctate, in front of prosternal process transversely wrinkled; punctures smaller than eye-facets, separated by one diameter in middle, becoming sparser laterally, interspaces smooth. Prosternal process broad, horizontal, punctate as pronotum, bordered at sides, widest near apex, apical margin shallowly concave in middle. Distances between pro-, meso- and metacoxae as $3: 4: 5$. Hypomera concave, markedly transversely wrinkled. Mesoventrite transversely vaulted, anterior margin completely bordered, anterior half impunctate, posterior one distinctly punctate, punctures smaller than eye-facets, separated by 1.0-1.5 diameters. Metaventrite almost flat in middle, flatly vaulted laterally, punctate like posterior half of mesoventrite. Discrimen absent. Suture between mesoand metaventrite very fine, obsolete, posterior intercoxal margin subtruncate. Mesocoxal lines somewhat S-shaped, median portion rectilinear and transverse, outer one almost rectilinear (safe its outer end), oblique, running posterolaterad to reach lateral margins of metaventrite at their midlength (Fig. 7). Axillary spaces and two anterior thirds of epipleura with small obsolete punctures separated by one diameter or less, interspaces reticulate, moderately shining. Posterior third of metepisterna separated by oblique raised ridge, with large, almost contiguous indistinct punctures, densely reticulate, dull. Abdominal ventrite I almost as long as metaventrite (ratio LMTV/LEV1 $=1.12$ ), punctures somewhat finer and shallower than on metaventrite. Metacoxal lines of two parts, inner one rectilinear, running posterolaterad from inner end of metacoxal cavity to posterior margin of ventrite, outer one transverse, arcuate. Abdominal ventrites II-IV equally long, markedly densely punctate; punctures smaller than eye-facets, separated by less than one diameter, interspaces smooth and shining. Hypopygium somewhat longer, broadly rounded apically.

Male genitalia. Median lobe of aedeagus nearly twice as long as wide, widest at apical third, slightly narrowed apically to distinctly incised apex; valves of phallotreme with acute tips directed anterolaterally (Fig. 9). Tegmen longer than wide, gradually dilated distad, lateral margins slightly arcuate; apical margin broad, subtruncate (Fig. 12). Distribution. Originally described from Old Calabar in Southern Nigeria (MurRAY 1867); currently also known from other countries in Africa south of the Sahara (see material examined). Thus far unknown from the southernmost part of Africa (Fig. 15).

\section{Prometopia hirta sp. nov.}

(Figs 5, 8)

Type locality. Zambia, Northwestern Province, between Chisasa and Solwezi.

Type material. HоLотуPE: + (NMPC), labelled: 'Zambia NW, E of / Chisasa, W of Solwezi / lgt. Snížek, 24.x.2008 [p] // HOLOTYPE $q$ / PROMETOPIA / hirta sp. nov. / Jelínek \& Hájek det. 2019 [p, red label]'. PARATYPES: 1 , labelled: 'ZAMBIA / CENTRAL PROVINCE / 15 km S KAPIRI MPOSHI / 30.11.2002 / A.KUDRNA JR. LGT. [p]' (ALBC); 3 우 , labelled: 'Zambia NW / $50 \mathrm{~km} \mathrm{E} \mathrm{of} \mathrm{Mwinilunga} \mathrm{/} \mathrm{lgt.}$ Snížek, 28.x.2008 [p]' (NMPC); 1 +, labelled: 'Zambia NW / 15 km E of Solwezi / 17.x.2008, lgt. Snížek [p]' (NMPC); 1 \%, labelled: 'TANZANIA-Kigoma pr. / $30 \mathrm{~km} \mathrm{NW} \mathrm{Uvinza/} \mathrm{S} 05^{\circ} 02^{\prime}$, E30 $22^{\prime}$ / 29.12.2006, $1500 \mathrm{~m} /$ Halada J. leg. [p]' (NMPC). All paratypes with the respective red printed paratype label.

Description. Female (holotype). Oblong oval, convex, moderately shining. Black, explanate sides of pronotum and elytra rusty, each elytron with two round orange spots, legs and antennae brown. Pubescence yellowish, long, semierect, sparse but conspicuous (Fig. 5).

Head narrower than anterior margin of pronotum $($ HEAW/WPR3 $=0.92)$, eyes subquadrangular (dorsal view) with short interfacetal setae, temples short, convergent. Anterior margin of clypeus truncate, not bordered; clypeus and frons separated by transverse impression between antennal insertion. Frons moderately convex with large reniform punctures, much larger than eye facets and bearing thick yellowish semierect setae on their anterior margin; interstriae smooth and shining with intermixed fine simple punctures bearing thin, shorter semidecumbent setae. Antennae shorter than width of head across eyes (ratio HEAW/ANLE $=1.18$ ), thin; antennal club occupying less than one third of antenna length (ratio ANLE/ $\mathrm{ANCL}=3.50$ ), oval, ratio $\mathrm{ANCL} / \mathrm{ANCW}=1.66$; scape subtriangular; antennomere III fairly as long as IV-VI combined; antennomere VIII as long as wide, not wider than preceding ones.

Pronotum transverse, widest posterior to midlength (ratio WPR2/LEPR = 2.09), more strongly narrowed anteriad than posteriad (ratio WPR1/WPR3 = 1.56). Anterior margin in middle truncate, not bordered; anterior angles sharp, prominent. Lateral margins arcuate, explanate (explanate sides twice as wide as antennal flagellum). Basal margin projecting into short subtruncate lobe in front of scutellum, besides it twice shallowly concave and bordered. Posterior angles obtuse, not prominent. Pronotal disc rather strongly transversely convex; median portion with punctures smaller than eye-facets and separated by more than one diameter; lateral thirds punctate like frons, with large reniform punctures intermixed with fine simple punctures. Long semierect yellowish setae intermixed with inconspicuous fine recumbent setae. Interspaces shining.

Scutellar shield transverse, rounded, mostly concealed by basal lobe of pronotum.

Elytra longer than their combined width (ratio LELY/ WELY $=1.04), 2.28 \times$ longer than pronotum, simultaneously rounded apically. Lateral margins narrowly explanate (nearly as wide as antennal flagellum), in basal half rectilinear and subparallel, in apical half arcuately converging towards apex; humeral angle obtusely angulate. Surface rather strongly transversely vaulted, humeral bulges indistinct. Punctures nearly equal in size to eye-facets, separated by about one diameter, intermixed with smaller ones; interspaces smooth and shining, becoming somewhat rugose at lateral margins. Pubescence apparently uniform, consisting of long semierect yellowish setae, shorter setae indistinct.

Pygidium broadly rounded, rugosely punctate.

Legs. Protibia $4.00 \times$ longer than wide, becoming gradually wider distad; outer edge finely crenulate, outer sub- 
apical angle with short acute spine. Metatibia $4.88 \times$ longer than wide, its inner margin broadly arcuate. Tarsomeres I-III of all tarsi simple, subcylindrical; tarsomere $\mathrm{V}$ as long as all preceding ones combined. Tarsal claws simple.

Ventral surface. Mentum 3.33× wider than long, punctures at sides as large as eye-facets, becoming smaller mesad. Postmentum shallowly concave, punctures equal in size to eye-facets, separated by one diameter or less, interspaces smooth and shining. Antennal furrows deep, partly concealed by extended S-shaped outer edges of postmentum. Genae posterior to eyes impunctate. Prosternum broadly transversely vaulted, sparsely punctate, punctures smaller than eye-facets and separated by ca. two diameters; interspaces smooth and shining. Prosternal process broad,
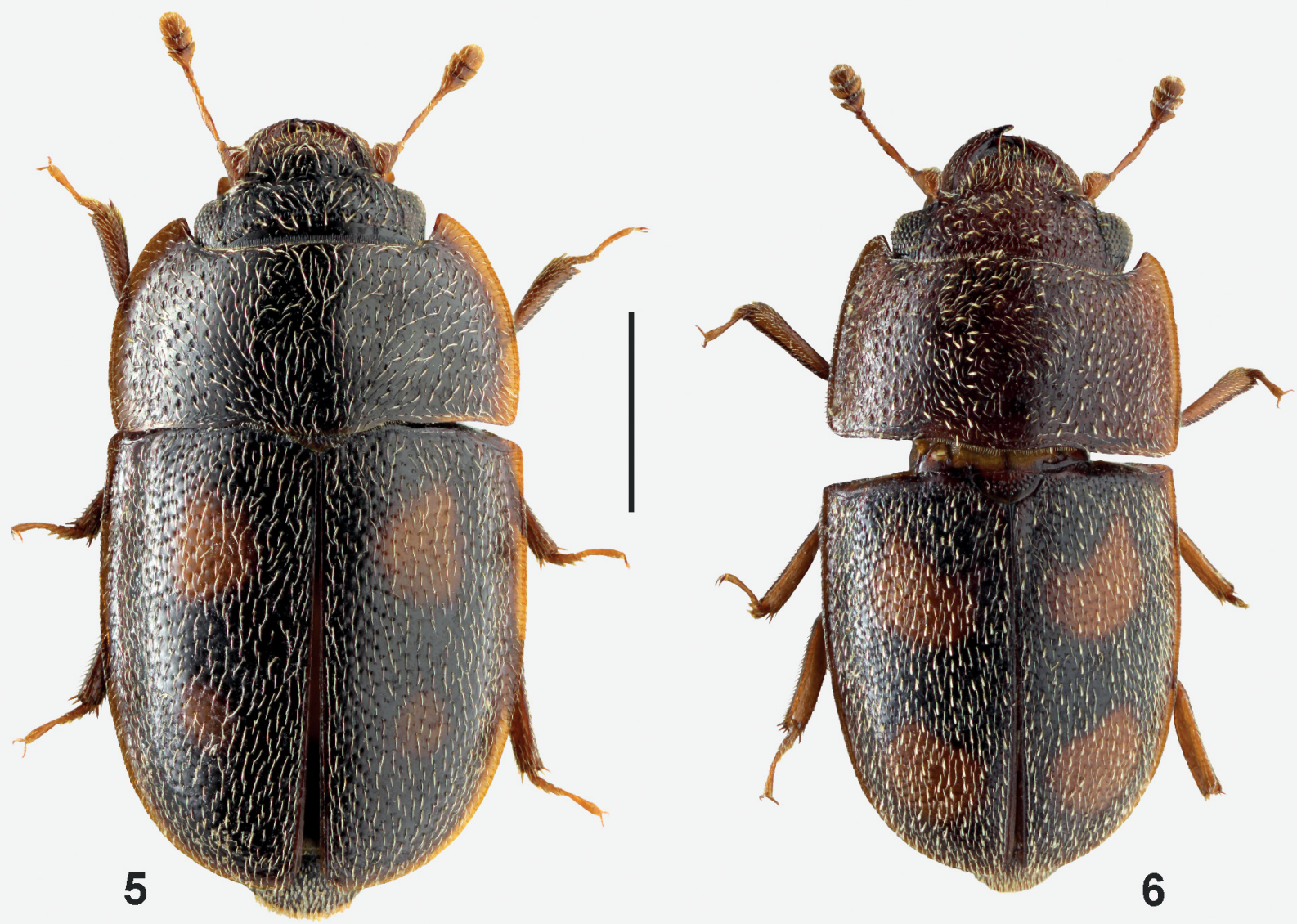

Figs 5-6. Dorsal habitus of Prometopia species. 5 - P. hirta sp. nov. (paratype, Zambia); 6 - P. gracilis Grouvelle, 1908 (India: Meghalaya). Scale bar $=1 \mathrm{~mm}$.
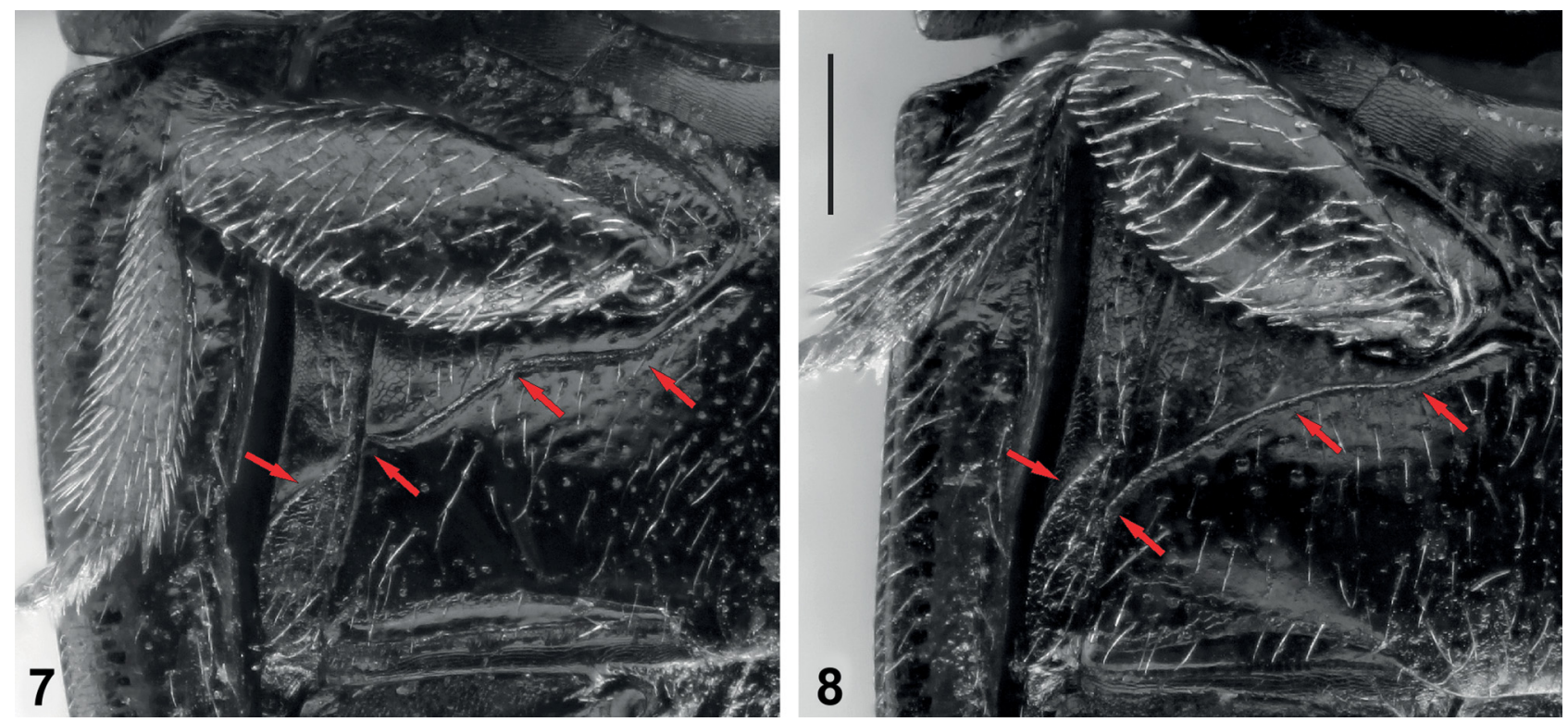

Figs 7-8. Meso- and metaventrite of Prometopia species. 7 - P. binotata Murray, 1867 (Zambia); 8 - P. hirta sp. nov. (paratype, Zambia). Scale bar $=$ $0.25 \mathrm{~mm}$. 
horizontal, bordered at sides, apical margin shallowly concave in middle, surface sparsely punctate like prosternum. Hypomera markedly transversely wrinkled. Mesoventrite transversely vaulted, anterior margin completely bordered, anterior half impunctate in middle, microscopically transversely strigose laterally, posterior half with punctures smaller than eye-facets and separated by more than one diameter; interspaces smooth and shining. Suture between meso- and metaventrite obsolete. Metaventrite flattened in middle, moderately convex laterally. Punctures somewhat smaller than those on mesoventrite, separated by several diameters, interspaces smooth and shining. Discrimen absent. Mesocoxal lines fluently shallowly S-shaped, reaching sternopleural suture posterior to midlength (Fig. 8). Metepisterna with obliquely transverse raised ridge reaching sternopleural suture before mesocoxal lines, punctation before and posterior to ridge equal. Abdominal ventrite I almost as long as mesoventrite (ratio LMEV/LVE1 = 1.10), sparsely punctate like mesoventrite. Metacoxal lines consisting (as in all Prometopiinae) of two parts: inner one rectilinear, running from inner corner of metacoxal cavity posterolaterad to posterior margin of ventrite I, and outer transverse, twice broken line, median portion of which is almost indistinct, running immediately at margin of coxal cavity; axillary space triangular, finely densely punctulate. Abdominal ventrites II-IV equally long, more densely punctate than ventrite I, punctures mostly separated by less than one diameter, interspaces with obsolete traces of reticulation. Hypopygium broadly rounded apically.

Male. Unknown.

Variation. The type series available does not display much variation.

Measurements. Body length 3.5-4.0 mm, width 1.9-2.1 $\mathrm{mm}$. Ratios HEAW/ANLE $=1.18-1.20$, HEAW/WPR3 = 0.89-0.95 (mean 0.91), WPR1/WPR2 = 0.94-0.96 (0.95), WPR1/WPR3 = 1.52-1.57 (1.54), WPR2/WPR3 = 1.58 1.67 (1.62), WPR2/LEPR = 2.03-2.18 (2.12), LELY/LEPR $=2.25-2.40$ (2.30), LELY/WELY $=1.03-1.07$ (1.05).

Differential diagnosis. Prometopia hirta sp. nov. differs from its Afrotropical congeners especially in its oblong body, the elytra being longer than their combined width with lateral margins rectilinear and subparallel in their

Table 1. Differential diagnosis of Prometopia hirta and P. gracilis.

\begin{tabular}{ll}
\hline \multicolumn{1}{c}{$\begin{array}{c}\text { Prometopia hirta } \text { sp. nov. } \\
\text { (Fig. 5) }\end{array}$} & \multicolumn{1}{c}{$\begin{array}{c}\text { Prometopia gracilis } \\
\text { (Fig. 6) }\end{array}$} \\
\hline $\begin{array}{l}\text { Pronotum widest posterior } \\
\text { to midlength, lateral margins } \\
\text { arcuate all along their length, } \\
\text { converging both anteriad and } \\
\text { posteriad. }\end{array}$ & $\begin{array}{l}\text { Pronotum widest at posterior an- } \\
\text { gles, lateral margins in basal half } \\
\text { rectilinear and subparallel. }\end{array}$ \\
\hline Humeral angles obtuse. & Humeral angles rectangular. \\
\hline $\begin{array}{l}\text { Lateral margins of elytra narrow- } \\
\text { ly explanate/canaliculate, nearly }\end{array}$ & $\begin{array}{l}\text { Lateral margins of elytra not } \\
\text { explanate. }\end{array}$ \\
as wide as antennal flagellum. & \\
\hline $\begin{array}{l}\text { Pubescence of elytra uniform, } \\
\text { consisting of conspicuous }\end{array}$ & $\begin{array}{l}\text { Pubescence of elytra double, } \\
\text { thick yellowish semierect setae } \\
\text { semirecumbent yellowish setae. } \\
\text { growing from background cove- } \\
\end{array}$ \\
& $\begin{array}{l}\text { red with dense semidecumbent } \\
\text { and markedly shorter setae. }\end{array}$ \\
\hline
\end{tabular}

basal half, and the dorsum being strongly transversely convex with conspicuous semierect yellowish pubescence (see also the above key).

With its habitus being oblong with a subparallel-sided convex body, and four orange spots on the elytra in addition to conspicuous semierect light pubescence, the new species most resembles Prometopia gracilis Grouvelle, 1908 from India, Nepal, Thailand and Sri Lanka. Both species can be distinguished by characters summarized in Table 1 .

Etymology. Latin adjective hirtus, - $a$, -um (= hairy, hirsute) refers to conspicuous pubescence distinguishing the new species from its African congeners.

Distribution. The new species is known so far from several localities in northwestern Zambia and western Tanzania; both areas are ca. $900 \mathrm{~km}$ apart (Fig. 15).

\section{Prometopia intermedia sp. nov.}

(Figs 2-3, 10, 13)

Type locality. Tanzania, Morogoro Province, Magombera Forest, $07^{\circ} 48^{\prime} 56.7^{\prime \prime} \mathrm{N}, 36^{\circ} 57^{\prime} 42.5^{\prime \prime} \mathrm{E}$

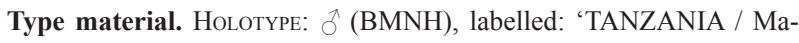
gombera Forest / S07 48'56.7”'E36 5 57'42.5” / 28.vii-1.viii.10 Light Trap / leg. Smith,R \& Takano,H [p] // BMNH€ / 2013-71 / 1460126 [p] // HOLOTYPE / / PROMETOPIA / intermedia sp. nov. / Jelínek \& Hájek det. 2019 [p, red label]'.

Additional material examined. TANZANIA: Morogoro province, 10 $\mathrm{km} \mathrm{N}$ of Mikumi, $7^{\circ} 19.6^{\prime} \mathrm{N} ; 36^{\circ} 57.4^{\prime} \mathrm{E}, 700$ m, 11.i.2007, F. Kantner lgt., 1 ( NMPC); see the description of female below.

Description. Male (holotype) (Fig. 2). Oval, moderately convex, moderately shining. Black, explanate sides of pronotum and elytra rusty, each elytron with one somewhat rhombic orange spot somewhat before its midlength, legs and antennae brown, pygidium brown, ventral surface reddish brown. Pubescence yellowish, recumbent, distinct.

Head narrower than anterior margin of pronotum (HEAW/WPR3 = 0.91), temples short, convergent. Clypeus and frons separated by transverse impression between antennal insertion. Clypeus flat with simple punctures smaller than eye-facets, separated by 1.0-1.5 diameters and bearing fine inconspicuous decumbent setae; interspaces smooth and shining. Anterior margin of clypeus subtruncate, not bordered. Frons moderately convex, punctate like clypeus with intermixed large flat reniform punctures nearly equal in size to eye-facets and bearing thick semidecumbent conspicuous setae. Antennae shorter than width of head across eyes (ratio HEAW/ANLE $=1.44$ ), antennal club oval (ratio ANCL/ANCW $=1.56$, occupying ca. one third of antenna length. Antennomere III almost as long as IV-VII combined; antennomeres IV and V subequal, nearly $1.5 \times$ longer than wide; antennomeres VI-VIII subequal, nearly as long as wide, antennomere VIII not wider than preceding ones.

Pronotum transverse, widest near posterior angles (ratio WPR2/LEPR $=2.31$ ), more strongly narrowed anteriad than posteriad (ratio WPR1/WPR3 = 1.32, WPR1/WPR2 $=0.91, \mathrm{WPR} 2 / \mathrm{WPR} 3=1.40$ ). Anterior margin in middle truncate, not bordered; anterior angles sharp, prominent. Lateral margins arcuate, explanate (explanate sides nearly as wide as antennal flagellum). Basal margin projecting into short subtruncate lobe in front of scutellum, besides it twice 
shallowly concave and finely bordered. Posterior angles obtuse, not prominent. Pronotal disc broadly vaulted, with small fine simple punctures separated by $1.5-2.0$ diameters and bearing fine inconspicuous decumbent setae reaching base of the following ones, intermixed with large shallow punctures bearing thicker semidecumbent yellowish setae. Interspaces smooth and shining.
Scutellar shield transverse, subtriangular, mostly concealed by basal lobe of pronotum. Its exposed apical portion impunctate.

Elytra nearly as long as their combined width (ratio LELY/WELY $=0.97), 2.23 \times$ longer than pronotum, simultaneously rounded apically. Lateral margins arcuate, slightly explanate. Dorsum more strongly transversely
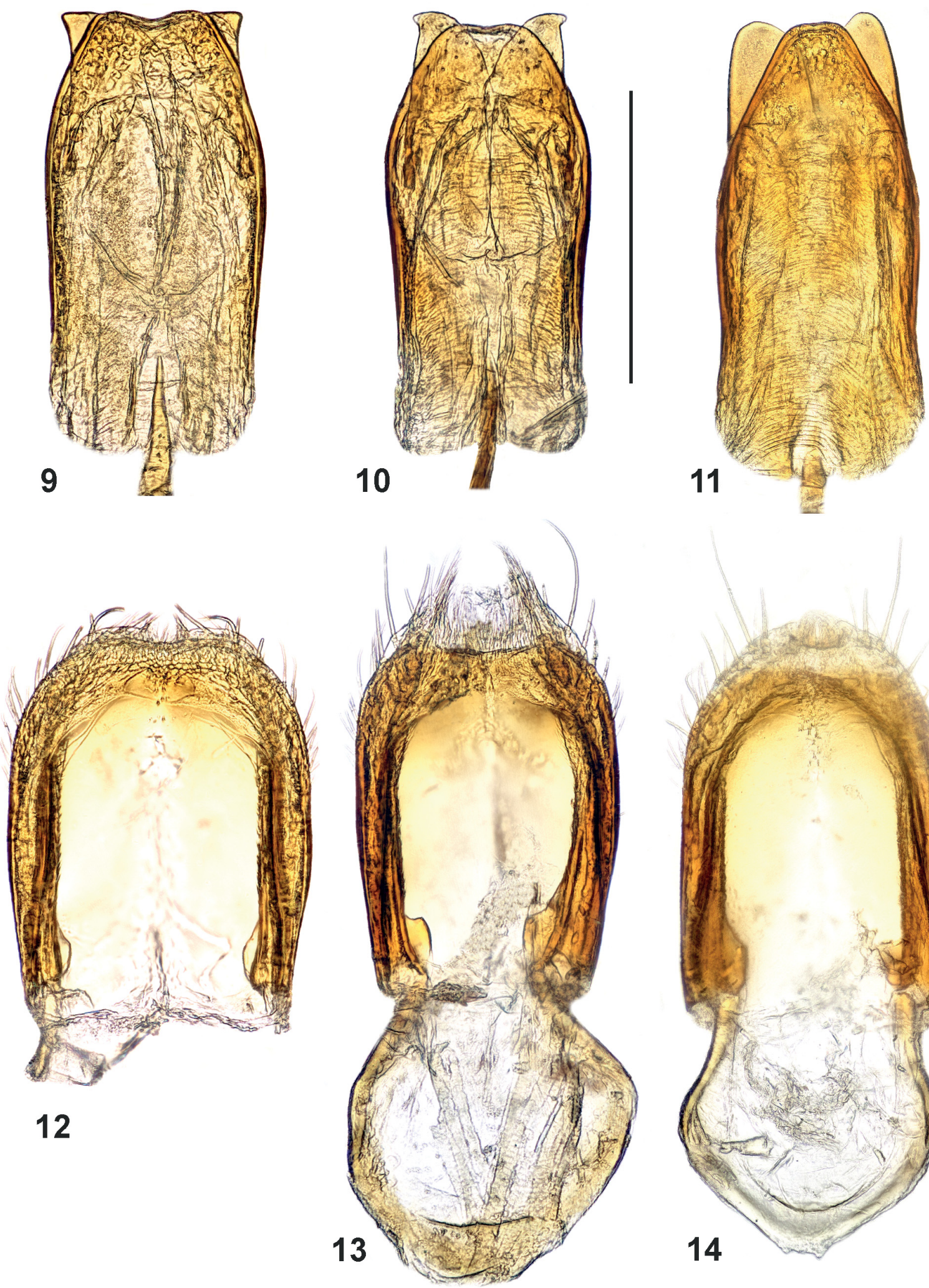

12

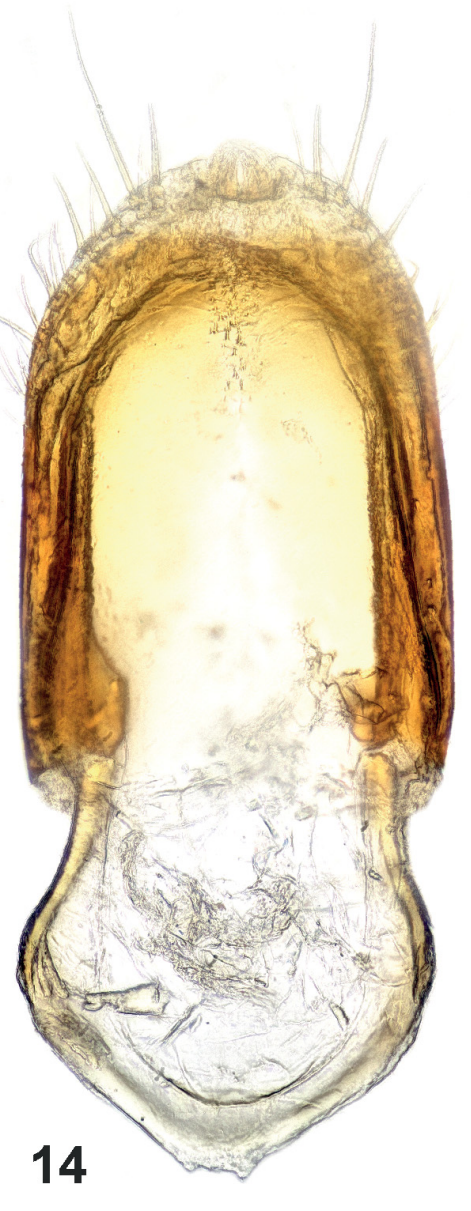

Figs 9-14. Male genitalia of Prometopia species. 9, 12 - P. binotata Murray, 1867 (Nigeria); 10, 13 - P. intermedia sp. nov.; 11,14 - P. quadrinotata Grouvelle, 1896 (RSA). 9-11 - median lobe of aedeagus; 12-14 - tegmen. Scale bar $=0.2 \mathrm{~mm}$. 
convex than pronotum. Punctures smaller than eye-facets, simple, in basal portion more pronounced and closer than those of pronotum, separated mostly by less than one diameter, becoming gradually finer and sparser posteriad, and larger and close, somewhat rugose laterally. Interspaces smooth and shining. Semidecumbent thin setae nearly reaching base of the following ones, intermixed with finer inconspicuous setae.

Pygidium broadly rounded, rugosely punctate and densely pubescent.

Legs. Femora oval, somewhat more than twice as long as wide. Protibia $3.50 \times$ longer than wide, widest at distal end, outer subapical angle subrectangular, not prominent. Meso- and metatibia ca. $4.28 \times$ and 5.10 $\times$ longer than wide respectively, flat, outer subapical angle rounded. Tarsomeres I-III of all tarsi simple, subcylindrical; tarsomere $\mathrm{V}$ longer than all preceding ones combined. Tarsal claws simple.

Ventral surface. Mentum transverse, $3.40 \times$ wider than long, punctures flat, larger than eye-facets, separated by less than one diameter, interspaces smooth and shining. Postmentum transversely concave (canaliculate) at base, punctures almost equal in size to eye-facets, separated by one diameter or less, interspaces smooth and shining. Antennal furrows deep, partly concealed by extended S-shaped outer edges of postmentum, converging posteriad. Genae posterior to eyes impunctate. Prosternum broadly transversely convex, in middle flat, anterior margin broadly finely bordered; punctures smaller than eye-facets, separated by 1-3 diameters in middle, becoming larger and closer laterally. Prosternal process broad, horizontal, bordered at sides, apical margin with broadly obtusely angulate emargination in middle, punctures in basal half shallow, smaller than eye-facets, separated by ca. two diameters, becoming smaller, deeper and closer in apical portion; interspaces smooth and shining. Hypomera transversely wrinkled and obsoletely punctate. Mesoventrite transversely vaulted and impunctate in front of mesocoxae, its posterior intercoxal portion almost flat, distinctly punctate, punctures markedly smaller than eye-facets, separated by 1.5-2.0 diameters, interspaces smooth and shining. Metaventrite almost flat in middle, punctate like intercoxal portion of mesoventrite, punctures becoming larger, deeper and closer (separated by one diameter or less) laterally. Discrimen absent. Outer portion of mesocoxal lines rectilinear, reaching sternopleural suture just posterior to oblique ridge on metepisterna. Metepisterna with raised oblique ridge nearly opposing mesocoxal lines, feebly reticulate and punctate like lateral portions of metaventrite, small subtriangular area anterior to raised ridge impunctate. Abdominal ventrite I as long as metaventrite, with analogous punctation. Metacoxal lines consisting of two parts: inner one rectilinear, running from inner corner of metacoxal cavity posterolaterad to posterior margin of ventrite I, and outer one transverse, asymmetrically arcuate, approaching posterior margin of metacoxal cavity at its outer half and vanishing at midlength of inner rectilinear part. Abdominal ventrites II-IV gradually shorter, punctate like ventrite I, but punctures are markedly closer, separated by one diameter or less.
Hypopygium broadly rounded, somewhat more coarsely and densely punctate.

Male genitalia. Median lobe of aedeagus nearly twice as long as wide, widest at apical third, narrowed towards narrow subtruncate apical margin; valves of phallotreme exceeding outline of lobe, with acute tips abruptly projecting outwards (Fig. 10). Tegmen longer than wide, gradually dilated distad, apical margin broad, shallowly concave (Fig. 13).

Female (Fig. 3). Single female available agrees in almost all details with the male holotype. It differs especially in the shape of pronotum, which is hardly narrowed towards posterior angles (ratio WPR1/WPR2 $=0.98$ ); lateral margins of pronotum in basal half rectilinear, slightly converging anteriad, moderately arcuate in anterior half (ratio WPR1/WPR3 = 1.34, WPR2/WPR3 = 1.35). Posterior angles rectangular. Larger punctures on median portion of pronotum less numerous and smaller, hardly equal in size to eye-facets. Pubescence more conspicuous. Body length $4.10 \mathrm{~mm}$, body width $2.40 \mathrm{~mm}$. Due to the extremely limited material examined it is difficult to evaluate the above differences. Because both specimens available were collected only about $50 \mathrm{~km}$ apart, we suppose that they are conspecific, but we prefer not to designate the female specimen as paratype.

Measurements. Body length 4.05-4.20 mm, width 2.40 $\mathrm{mm}$. Ratio WPR1/WPR2 $=0.91-0.98, \mathrm{WPR} 1 / \mathrm{WPR} 3=$ 1.32-1.34, WPR2/WPR3 = 1.35-1.40, WPR2/LEPR = 2.31-2.37, LELY/WELY $=0.93-0.97$.

Differential diagnosis. With its colour pattern Prometopia intermedia sp. nov. resembles $P$. binotata, from which it differs in having long double pubescence, resembling that of $P$. quadrinotata. However, it differs from the latter species in the colour pattern as well as in the shape of male genitalia with abruptly hooked tips of the valves of the phallotreme (Fig. 10).

Etymology. Latin adjective intermedius, - $a$, -um (= intermediate) refers to intermediate morphology of the new species between $P$. binotata and P. quadrinotata.

Distribution. The new species is known so far from two close localities in southeastern Tanzania (Fig. 15).

\section{Prometopia quadrinotata Grouvelle, 1896} (Figs 4, 11, 14)

Prometopia quadrinotata Grouvelle, 1896: 72 (original description); Grouvelle (1913): 132 (catalogue).

Material examined. BURKINA FASSO: Upper Volta, Boho Diulasso, i.-iii.1975, Politzer lgt., 1 spec. (NMPC). DEMOCRATIC REPUBLIC OF THE KONGO: Zaire, Kole Kasai, 1 spec. (NMPC, ex coll. O. Marek); N.P.G. (Garamba), Mission de Saeger, II/gd/4, 28.iv.1952, H.DeSaeger3384, 3 spec.; same data, but 26.v.1952, H.DeSaeger3519, 1 spec.; II/dd/9, 7.vii.1952, H.DeSaeger3744, 4 spec.; Morubia/9, 12.iii.1952, H.DeSaeger3186, 1 spec.; Utukuru/9, 26.vii.1952, H.DeSaeger3823, 1 spec. (all NMPC). EQUATORIAL GUINEA: Guinea Espaňol, Evinayong, Dr. Báguena lgt., 17 spec.; Mikomeseng, Dr. Báguena lgt., 4 spec.; Rio Benito, Dr. Báguena lgt., 5 spec.; Bata, 2 spec. (all NMPC, ex coll. O. Marek). GUINEA: French Guinea, Kindia, iv.1954, 1 spec. (NMPC). SOUTH AFRICA: KwaZulu-Natal, Jozini, Tembe, Elephant Park env. (W border), 22.i.2003, V. Křivan lgt., 1 spec. (NMPC); KwaZulu-Natal, SW Magudu, $27^{\circ} 24^{\prime}$ S, $31^{\circ} 35^{\prime}$ E, 4.- $-5 . i .2009$, P. Schüle lgt., 3 spec. (SMNS). TANZANIA: Ruvuma province, Mbinga Range, Ndongosi vill., 10.i.2007, K. Angelus lgt., 1 spec. (ALBC). 


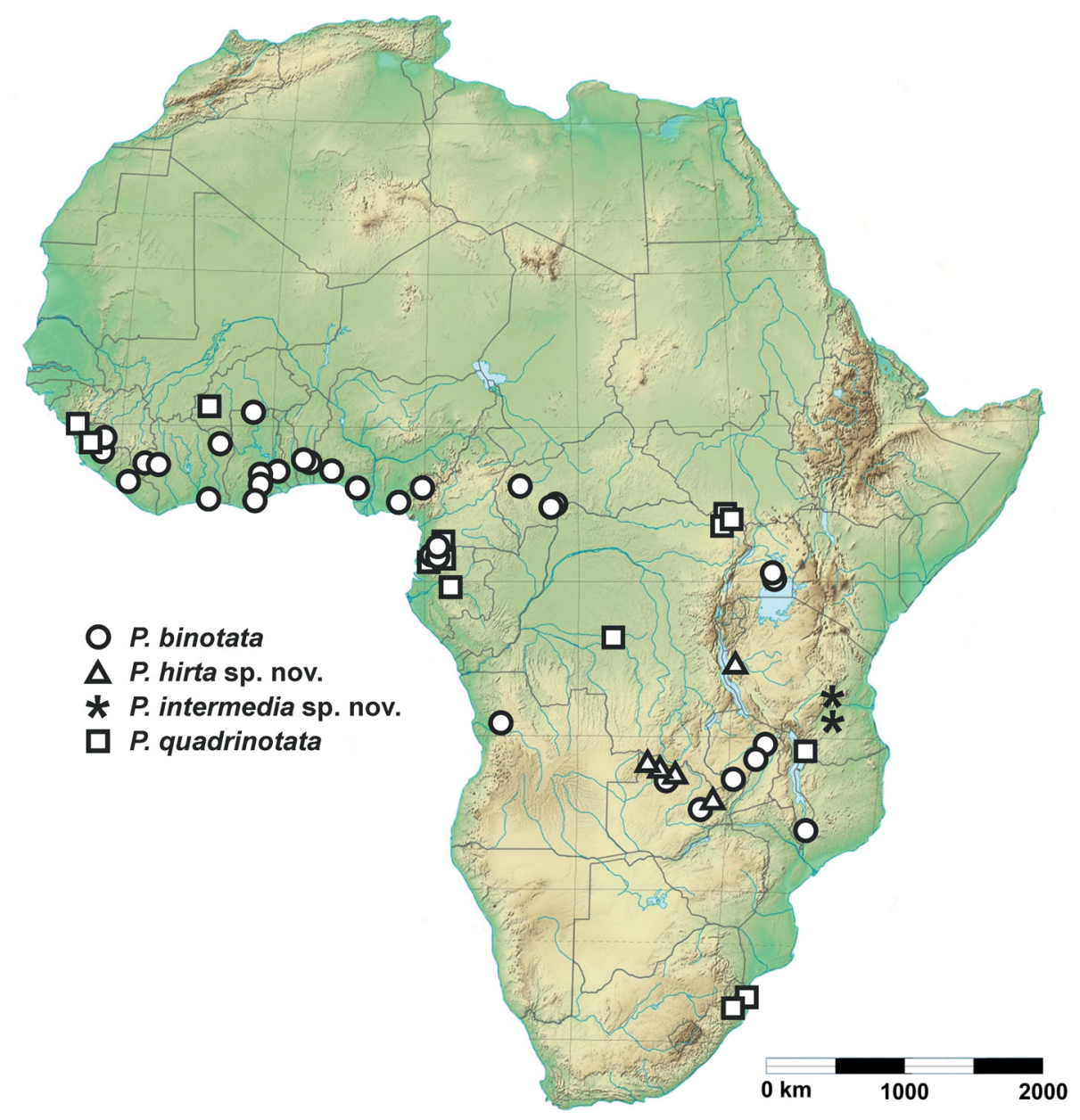

Fig. 15. Map of distribution of Prometopia species in Africa.

Redescription. Oval, moderately convex, brown to black-brown, each elytron with two, more or less, round orange spots of variable size, one situated in inner anterior quarter, second in apical half of elytron; explanate sides of pronotum and elytra, legs, antennae and mouth parts rusty; ventral side red-brown, metaventrite sometimes darker, piceous. Pubescence thin, yellowish, semidecumbent (Fig. 4). Body length 3.0-4.3 mm, width $1.8-2.7 \mathrm{~mm}$.

Head across eyes narrower than anterior pronotal margin. Eyes coarsely facetted, widest in anterior portion, gradually narrowed posteriad, with short indistinct interfacetal setae. Temples short, convergent. Frons separated from clypeus by transverse impression; clypeus truncate, not bordered, forming an obtuse angle with frons. Punctures of frons smaller than eye-facets, separated by less than one diameter, on clypeus sparser, separated by one diameter, on frons intermixed with umbillicate punctures larger than eye-facets, which moreover, form a series along occipital sulcus; interspaces smooth and shining. Antennae shorter than width of head across eyes (ratio HEAW/ANLE = 1.50 ), slender, antennal club occupying less than one third of antenna length (ratio ANLE/ANCL $=2.88$ ), oval, ca. $1.66 \times$ longer than wide.

Pronotum transverse (ratio WPR2/LEPR $=2.35-2.58$ ), widest at base (ratio WPR1/WPR2 $=0.97-0.98$ ), gradu- ally narrowed anteriad (ratio WPR1/WPR3 = 1.48-1.66, WPR2/WPR3 = 1.53-1.69). Anterior margin not bordered, truncate; anterior angles acute, strongly prominent. Basal margin with subtruncate prescutellar lobe, besides it obliquely subtruncate, completely (however indistinctly so in middle) bordered; basal rim with series of fine punctures. Posterior angles feebly obtuse, not prominent. Lateral margins arcuate, gradually converging anteriad; sides explanate, as wide as length of scape. Punctures generally smaller than eye-facets, but of varying size, separated by 1.0-1.5 diameters; at sides and at base besides scutellum intermixed with large umbillicate punctures separated by 0.5-1.0 diameters. Semirecumbent thin yellowish setae fairly reaching base of the following ones, sparse but distinct, intermixed with thin darker recumbent, hardly distinct, setae.

Scutellar shield transverse, almost semicircular, punctate, with broad impunctate border; punctures equal in size to smallest ones on pronotum, separated by ca. one diameter.

Elytra oval, regularly convex, widest before their midlength, almost as long as their combined width, ratio WELY/LELY $=1.01-1.06$ (mean 1.03). Lateral margins arcuate, gradually converging posteriad, slightly so anteriad; sides narrowly explanate, as wide as antennal 
flagellum. Humeral angle obtuse. Humeral bulges low, inconspicuous. Sutural line absent. Punctures small, more uniform than those on pronotum, separated by ca. 1.0-1.5 diameters on disc, becoming larger, closer and rugose at sides. Pubescence like on pronotum.

Legs. Femora oval, somewhat more than twice as long as wide. Protibia ca. $4.25 \times$ longer than wide, widest at distal end, outer subapical angle subrectangular, not prominent. Meso- and metatibia ca. $4.60 \times$ and $4.80 \times$ longer than wide respectively, flat, outer subapical angle rounded.

Ventral surface as in P. binotata. Punctures on postmentum almost equal in size to eye-facets, shallow, oval, separated by less than one diameter, interspaces smooth and shining. Prosternum without wrinkles in front of prosternal process. Punctures of metaventrite somewhat smaller and shallower than in P. binotata.

Male genitalia. Median lobe of aedeagus more than twice as long as wide, widest at midlength, in apical portion strongly narrowed towards truncate apex, valves of phallotreme rounded apically (Fig. 11). Tegmen longer than wide, parallel-sided in basal half; apical margin broad, rounded, medially shallowly incised (Fig. 14).

Distribution. Originally described from Gabon, Sierra Leone and Ethiopia (Grouvelle 1896). Currently also known from other countries of Subsaharan Africa, as far southwards as Maputaland, RSA (see material examined and Fig. 15).

\section{Conclusions}

Four species of the genus Prometopia are currently known from Africa south of Sahara. Close relationship between the two previously known species, Prometopia binotata, $P$. quadrinotata, and the newly described $P$. intermedia sp. nov. is suggested by conformity in the shape of the pronotum and elytra, pubescence, a comparatively long mentum, and configuration of the mesocoxal lines and metepisterna. At the same time, they differ in all above characters from those exhibited in P. hirta sp. nov. On the contrary, the overall similarity of $P$. hirta sp. nov. with the compared Oriental $P$. gracilis does not necessarily indicate a close relationship between the two species.

\section{Acknowledgements}

We are obliged to Andrzej Lasoń (Bialystok, Poland), Maxwell V. L. Barclay and Michael Geiser (BMNH), and Wolfgang Schawaller (SMNS) for loan of the material. We are grateful to Alain Coache (CEREB) for sharing his data, and to Gareth S. Powell (Brigham Young University, Provo, Utah, USA) for valuable comments on the manuscript. The present work was supported by the Ministry of Culture of the Czech Republic (DKRVO 2019-2023/5.I.b, National Museum, 00023272).

\section{References}

AUDISIO P. \& JELÍNEK J. 1993: Two new genera of Nitidulidae from the Oriental Region, with notes on phylogeny of the "Axyroid-group" (Coleoptera, Nitidulidae, Nitidulinae). Revue Suisse de Zoologie 100: $405-423$.
BOUCHARD P., BOUSQUET Y., DAVIES A. E., ALONSO-ZARAZAGA M. A., LAWRENCE J. F., LYAL C. H. C., NEWTONA. F., REID C. A. M., SCHMITT M., ŚLIPIŃSKI S. A. \& SMITH A. B. T. 2011: Family-group names in Coleoptera (Insecta). ZooKeys 88: 1-972.

BÖVING A. G. \& CRAIGHEAD F. C. 1931: An illustrated synopsis of the principal larval forms of the order Coleoptera. Entomologica Americana, New Series 11 [1930-1931]: 1-351.

CLINE A. R., SMITH T. R., MILLER K., MOULTON M., WHITING M. \& AUDISIO P. 2014: Molecular phylogeny of Nitidulidae: assessment of subfamilial and tribal classification and formalization of the family Cybocephalidae (Coleoptera: Cucujoidea). Systematic Entomology 39: 758-772.

DASGUPTA J., PAL T. K. \& HEGDE V. D. 2015: Review of the Megauchenia Macleay of India (Coleoptera: Nitidulidae: Prometopinae). Zootaxa 4058: 551-560.

GIMMEL M. L. \& FERRO M. L. 2018: General overview of saproxylic Coleoptera. Pp. 51-128. In: ULYSHEN M. D. (ed.): Saproxylic insects. Diversity, ecology and conservation. Zoological Monographs 1: i-ix + 1-904.

GROUVELLE A. 1896: Descriptions de clavicornes d'Afrique et de Madagascar. Annales de la Société Entomologique de France 65: 71-94.

GROUVELLE A. 1913: Byturidae, Nitidulidae: 1. Cateretinae, 2. Meligethinae, 3. Carpophilinae, 4. Nitidulinae, 5. Cryptarchinae, 6. Cybocephalinae. In: SCHENKLING S. (ed.): Coleopterorum Catalogus. Pars 56. W. Junk, Berlin, 223 pp.

ICZN 1999: International Code of Zoological Nomenclature. Fourth edition. The International Trust for Zoological Nomenclature, London, $306 \mathrm{pp}$.

JELÍNEK J. \& AUDISIO P. 2007: Nitidulidae. Pp. 459-491. In: LÖBL I. $\&$ SMETANAA. (eds): Catalogue of Palaearctic Coleoptera. Volume 4. Elateroidea - Derodontoidea - Bostrichoidea - Lymexyloidea Cleroidea - Cucujoidea. Apollo Books, Stenstrup, 935 pp.

JELÍNEK J. \& HÁJEK J. 2018: The first species of the genus Platychora Erichson, 1843 from the Australian Region (Coleoptera, Nitidulidae, Prometopinae). Entomologische Blätter für Biologie und Systematik der Käfer 114: 255-261.

KIREJTSHUK A. G. 2005: On the fauna of Nitidulidae (Insecta, Coleoptera) from Taiwan with some taxonomical notes. Annales Historico-Naturales Musei Nationalis Hungarici 97: 51-113.

KIREJTSHUK A. G. 2008: A current generic classification of sap beetles (Coleoptera, Nitidulidae). Zoosystematica Rossica 17: 107-122.

KIREJTSHUK A. G. \& MANTIČ M. 2015: On systematics of the subfamily Cybocephalinae (Coleoptera: Nitidulidae) with description of new species and generic taxa. Proceedings of the Zoological Institute RAS 319: 196-214.

LAWRENCE J. F. 2016: 2. Classification (families \& subfamilies). Pp. 13-22. In: BEUTEL R. G. \& LESCHEN R. A. B. (eds): Handbook of Zoology. Arthropoda: Insecta. Coleoptera, Beetles. Volume 1: Morphology and Systematics (Archostemata, Adephaga, Myxophaga, Polyphaga partim). $2^{\text {nd }}$ edition. Walter de Gruyter, Berlin/Boston, $\mathrm{xvii}+684 \mathrm{pp}$.

LEE M. H., LEE Seunghyun \& LEE Seunghwan 2018: New record of subfamily Prometopinae (Coleoptera: Cucujoidea: Nitidulidae) in Korea. Journal of Asia-Pacific Entomology 21: 239-243.

LUCAS R. 1920: Catalogus alphabeticus generum et subgenerum coleopterorum orbis terrarum totius. Berlin, $696 \mathrm{pp}$.

MURRAY A. 1867: List of Coleoptera received from Old Calabar, on the West coast of Africa. The Annals and Magazine of Natural History, including Zoology, Botany, and Geology, Third Series 19: 167-180. MURRAY A. 1878: List of Coleoptera from Old Calabar, on the West coast of Africa. Taylor and Francis, London, $221 \mathrm{pp}+6 \mathrm{pls}$. 
